\title{
The approach of a vortex pair to a plane surface in inviscid fluid
}

\author{
By P. G. SAFFMAN \\ Department of Applied Mathematics, California Insitute of Technology, \\ Pasadena
}

(Received 18 September 1978)

It is shown that a symmetrical vortex pair consisting of equal and opposite vortices approaching a plane wall at right angles must approach the wall monotonically in the absence of viscous effects. An approximate calculation is carried out for uniform vortices in which the vortices are assumed to be deformed into ellipses whose axis ratio is determined by the local rate of strain according to the results of Moore \& Saffman (1971).

\section{Introduction}

The behaviour of a vortex pair in a ground effect is of some interest for aerodynamic and environmental reasons. The simplest theoretical treatment models the vortex pair by two rectilinear line vortices of equal and opposite circulation at the same height above an infinite plane. Each vortex moves in the velocity field induced by the other and the images in the plane. The trajectories are easily computed (see Lamb 1932, p. 223) and are found to be the curves

$$
x_{0}^{2}\left(x^{2}+y^{2}\right)=x^{2} y^{2},
$$

where $x=0$ is the plane of symmetry, $y=0$ is the wall, and $2 x_{0}$ is the initial separation of the vortices when infinitely far from the plane. According to this elementary solution the vortices approach the wall monotonically as they separate from one another, the final asymptotic distance from the wall being $x_{0}$.

Dee \& Nicholas (1968) reported on flight test observations of trailing vortices produced by an aircraft flying close to the ground. They found moderate agreement between the observed trajectories and those predicted by the elementary theory when allowance is made for additional horizontal displacement produced by a cross-wind. Harvey \& Perry (1971) noted, however, that the theory and the observations differed in one noteworthy respect. In many of the tests, the vortices rose again after having approached the ground, i.e. they appeared to bounce. Harvey \& Perry carried out some wind-tunnel experiments on a single vortex shed by a half-span wing over a moving floor and concluded that the cause of the vortex bounce is separation of the boundary layer on the ground underneath the vortex. They argued that boundary-layer separation produces a secondary vortex whose development slows down the horizontal motion of the primary vortex and makes it rise. It was reported that in some configurations the horizontal motion of the primary vortex could be reversed.

Recently and in ignorance of the earlier work, Barker \& Crow (1977) observed rebounding for a vortex pair, generated in water, approaching either the free surface or a horizontal rigid plane immersed in the water. They claimed that the phenomenon 
can be attributed to the effect of finite core size, which is of course neglected in the elementary theory. However, they gave no evidence to support their contention apart from reference to unpublished numerical simulations and an unsubstantiated statement that core deformation would lead to unsteadiness that could explain the effect.

We shall present below simple arguments to show that in the framework of an inviscid theory, the rebounding cannot be explained by finite core size and that the velocity of the vortices towards the wall cannot change sign. We shall also calculate the paths and shapes of the vortices by means of an extension of an approximation introduced by Moore \& Saffman (1975) which was used quite successfully by them and others (e.g. Bilanin, Teske \& Williamson 1977) in similar contexts.

The implication of these calculations is that the explanation of rebounding given by Harvey \& Perry is the correct one, at least for rigid walls. However, the phenomenon was also observed by Barker \& Crow when the vortices approached a free surface. Since the boundary layer at a free surface is much weaker than that at a rigid boundary (and is in a sense continually separating) it is most unlikely that secondary vortices would be produced in this case as required by Harvey \& Perry's explanation. Unless surface contamination in Barker \& Crow's experiment had the effect of making the free surface behave like a rigid boundary with respect to slip, their observations that the nature of the boundary had little effect upon the gross properties of the vortex motion would suggest an inviscid explanation. Since an inviscid explanation dose not exist, the observations on rebounding at a free surface present an unsolved problem.

\section{Theoretical considerations}

From symmetry, it is sufficient to consider the vorticity $\omega$ in the positive quandrant bounded by the wall $(y=0)$ and the axis of symmetry $(x=0)$. The motion is twodimensional with velocity components $(u, v)$. The total vorticity in the quadrant is given by

$$
\Gamma=\int_{0}^{\infty} \int_{0}^{\infty} \omega d x d y
$$

The circulation $\Gamma$ is conserved when viscosity is neglected. Viscous effects would produce vorticity at the wall and cause diffusion of vorticity across the axis of symmetry.

The components $\left(I_{x}, I_{y}\right)$ of hydrodynamic impulse of the vorticity are

$$
I_{x}=\int_{0}^{\infty} \int_{0}^{\infty} y \omega d x d y, \quad I_{y}=-\int_{0}^{\infty} \int_{0}^{\infty} x \omega d x d y .
$$

The co-ordinates $(\bar{x}, \bar{y})$ of the vorticity centroid are

$$
\bar{x}=-I_{y} / \Gamma, \quad \bar{y}=I_{x} / \Gamma .
$$

It follows from Euler equations for incompressible inviscid fluid that

$$
\frac{d I_{x}}{d t}=-\int_{0}^{\infty} \int_{0}^{\infty}\left\{\frac{\partial}{\partial x}(u \omega y)+\frac{\partial}{\partial y}(v \omega y)-v \omega\right\} d x d y
$$

Integrating by parts, using the kinematic boundary conditions

$$
u=0 \quad \text { on } \quad x=0, \quad v=0 \quad \text { on } \quad y=0,
$$


which follow from symmetry, and using (2.3) to replace $I_{x}$ by $\bar{y}$, we obtain

Similarly,

$$
\frac{d \bar{y}}{d t}=-\frac{1}{2 \Gamma} \int_{0}^{\infty} v_{0}^{2} d y
$$

\section{Here}

$$
\frac{d \bar{x}}{d t}=\frac{1}{2 \Gamma} \int_{0}^{\infty} u_{0}^{2} d x
$$

Suppose that the vortex pair is initially at a large distance from the wall $x=0$. Then (2.6) predicts that $\bar{y}$ decreases at a rate depending upon the velocity field on the axis of symmetry. As the pair approaches the wall, the vortices begin to separate under the action of the image vorticity in the wall. This is also shown by (2.7), according to which $\bar{x}$ increases when $u_{0}$ is not negligibly small on the wall. Eventually, the vortices will be so far apart that $v_{0}$ is negligible. The centroids of the vortices will then have reached an asymptotic distance from the wall and continue parallel to it. Since the right-hand side of (2.6) is always negative, inviscid dynamics predicts that the vorticity centroids approach the wall monotonically, however large the vortices or their deformation may be, and asymptote to a line parallel to the wall.

We cannot assert that the final motion of each vortex is steady. It is possible that the final state is a periodic or aperiodic oscillation. However, if the final motion is steady, then the time reversibility of the Euler equations ensures that the final distance from the wall is equal to one-half the initial separation.

We should mention the possibility that the rebounding of the vortices is apparent and not real. Hooker (1936) claimed that the observed increasing spacing ratios of Kármán vortex sheets is due to a divergence between the centroid of vorticity and the centre of rotation of a vortex as it spreads. The centre of rotation is the instantaneous stagnation point of the flow as measured by a given observer, depends of course on the observer's speed, and would be indentified as the natural centre of the vortex by common flow-visualization techniques. Thus deformation of a vortex as it approached the wall might lead to an apparent movement of the vortex away from the wall. However, we shall estimate this effect in our approximate calculation to be described in the next section and show that it does not occur.

\section{An approximate calculation for uniform vortices}

The deformation of the vortices and its effect on their trajectories can be estimated simply if we assume that the vortices are elliptical with axis ratio and orientation given by the theory of Moore \& Saffman (1971) for steady uniform vortices in uniform steady straining fields. We apply this theory by assuming (cf. Moore \& Saffman 1975) that the appropriate value of the uniform straining field is the rate of strain at the centre of the vortex due to the other vortex and the images in the plane. The configuration of the vortex pair and its image is then as shown in figure 1 . The centre of the vortex in the first quadrant is at $(\bar{x}, \bar{y})$. The semi-major and semi-minor axes have length $a$ and $b$, respectively and the major axis is inclined at angle $\theta$ to the $x$ axis.

We require the velocity and rates of strain induced at $(\bar{x}, \bar{y})$ by the other three vortices. Consider a uniform elliptical vortex, of strength $\kappa$, bounded by

$$
X^{2} / a^{2}+Y^{2} / b^{2}=1 \text {. }
$$



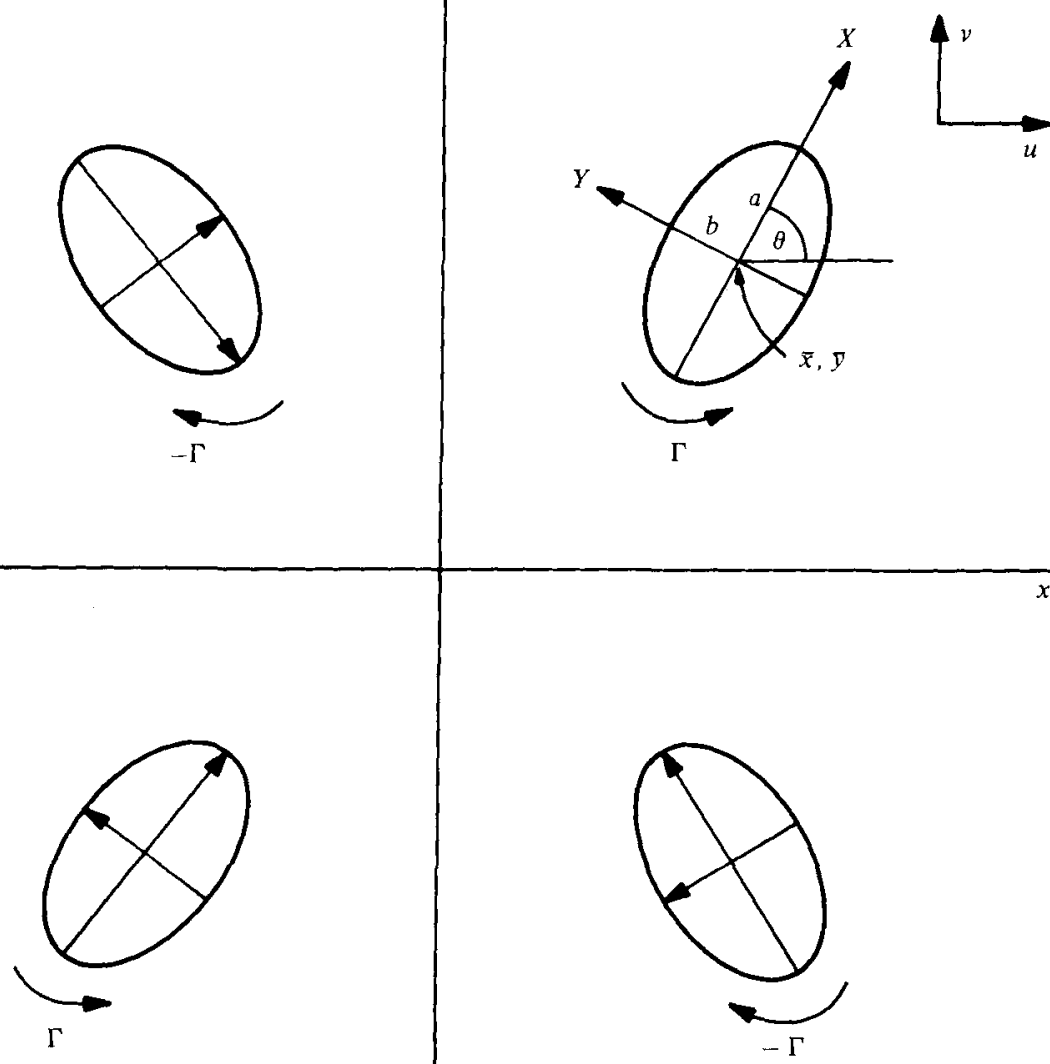

Figure 1. Configuration of the vortex pair and its image in the wall $y=0$.

An elementary calculation shows that the complex potential $w(Z)(Z=X+i Y)$ outside the ellipse is

$$
W=-i \kappa / 2 \pi\left(\zeta+\frac{1}{2} e^{-2 \zeta}\right),
$$

where

$$
Z=c \cosh \zeta, \quad c^{2}=a^{2}-b^{2}, \quad \zeta=\xi+i \eta
$$

The complex velocity at the point $(X, Y)$ relative to the axes of the ellipse is

$$
U-i V=\frac{d W}{d \zeta} / \frac{d Z}{d \zeta}=-\frac{i \kappa}{\pi c} e^{-\zeta}
$$

Denote the rate-of-strain tensor $e_{i j}=\frac{1}{2}\left(\partial u_{i} / \partial x_{j}+\partial u_{j} / \partial x_{i}\right)$ at $(X, Y)$ by

$$
\left(\begin{array}{rr}
A & B \\
B & -A
\end{array}\right)
$$

Then

$$
A-i B=\left(d^{2} w / d z^{2}\right)=\left(i \kappa / \pi c^{2}\right) e^{-\zeta} \operatorname{cosech} \zeta .
$$

Relative to axes $O x y$ which make an angle $\phi$ with those of the ellipse, the velocity components $(u, v)$ and corresponding components $(\alpha, \beta)$ of the rate-of-strain tensor are given by

$$
u-i v=(-i \kappa / \pi c) e^{-\zeta} e^{i \phi}, \quad \alpha-i \beta=\left(i \kappa / \pi c^{2}\right) e^{-\zeta} e^{2 i \phi} \operatorname{cosech} \zeta .
$$




\begin{tabular}{cclllr}
\hline Quadrant & Strength & \multicolumn{1}{c}{$X$} & \multicolumn{1}{c}{$Y$} & $\theta$ \\
2 & $-\Gamma$ & $2 \bar{x} \cos \theta$ & $2 \bar{x} \sin \theta$ & $\theta$ \\
3 & $\Gamma$ & $2\left(\bar{x}^{2}+\bar{y}^{2}\right)^{\frac{1}{2}} \cos \left(\theta-\tan ^{-1} \bar{y} / \bar{x}\right)$ & $-2\left(\bar{x}^{2}+\bar{y}^{2}\right)^{\frac{1}{2}} \sin \left(\theta-\tan ^{-1} \bar{y} / \bar{x}\right)$ & $-\theta$ \\
4 & $-\Gamma$ & $2 \bar{y} \sin \theta$ & $-2 \bar{y} \cos \theta$ & $\theta-\pi$
\end{tabular}

TABLE 1. Parameters for the calculation of the velocity and rate-of-strain at the centre of the vortex in the first quadrant.

The values of $\kappa, X, Y$ and $\phi$ for the contribution at $(\bar{x}, \bar{y})$ by the vortices in the second, third and fourth quadrants are listed in table 1.

Substitution of these values into (3.7) and summing over the three vortices gives the velocity $(\bar{u}, \bar{v})$ and rate of strain $(\bar{\alpha}, \bar{\beta})$ at the centre of the vortex. The motion and shape of the vortices are determined by the equations

$$
\begin{gathered}
d \bar{x} / d t=\bar{u}, \quad d \bar{y} / d t=\bar{v} \\
\theta=\frac{1}{2} \tan ^{-1} \frac{\bar{\beta}}{\bar{\alpha}}+\frac{\pi}{4}, \quad \frac{\epsilon(\epsilon-1)}{\left(\epsilon^{2}+1\right)(\epsilon+1)}=\frac{\pi a b}{\Gamma}\left(\bar{\alpha}^{2}+\bar{\beta}^{2}\right)^{\frac{1}{2}}
\end{gathered}
$$

Here $\epsilon=a / b$ is the axis ratio of the ellipse. Equation (3.8) assumes that the vortex moves with the velocity at its centre. Equation (3.9) is the result of Moore \& Saffman (1971) for the shape of a uniform vortex in a uniform straining field. The size of the vortex is conserved during the motion; hence

$$
a b=R^{2},
$$

where $\pi R^{2}$ is the area of the vortex. Note that (3.9) has no solution if

$$
(\pi a b / \Gamma)\left(\bar{\alpha}^{2}+\bar{\beta}^{2}\right)^{\frac{1}{2}}>0 \cdot 15 .
$$

If this occurs, the vortex is expected to disintegrate.

When $\bar{y}=\infty$, we have the case of a single vortex pair. Then $\theta=\frac{1}{2} \pi$, the major axes of the pair are parallel, and the equation for $\epsilon$ reduces to

$$
\frac{(\epsilon-1)^{2}}{\epsilon^{2}+1}=1-\left[1+\frac{R^{2}}{4 x^{2}}\left(\epsilon-\frac{1}{\epsilon}\right)\right]^{-\frac{1}{2}} \text {. }
$$

The solution of this equation giving $\epsilon$ as a function of $R / \bar{x}$ is shown in figure 2 , together with the values of $b / \bar{x}$ and $-4 \pi \bar{x} \bar{v} / \Gamma$. Note that solutions exist for all $R / \bar{x}$. Further, $b / \bar{x} \rightarrow 1$ as $R / \bar{x} \rightarrow \infty$, i.e. the vortices become long and elongated and approach one another more closely as the distance between their centres tends to zero. The speed of the pair is less than the velocity of two point vortices at their centres. This behaviour is qualitatively similar to that of the exact solution for two hollow vortices (Pocklington 1894). Notice that the condition (3.11) is never violated, so this simple theory predicts that a vortex pair never disintegrates however close the vortices are. Of course, the theory may not be accurate for values of $a / b$ much larger than one.

We can estimate the position of the centre of rotation by finding the point at which the velocity produced by the vortex in the first quadrant is equal and opposite to the velocity of its centre. Inside the ellipse, the stream function in Cartesian coordinates of the velocity relative to the centre is

$$
\psi=\left(\Gamma / 2 \pi a b c^{2}\right)\left[X^{2}\left(b^{2}-a b\right)-Y^{2}\left(a^{2}-a b\right)\right] .
$$




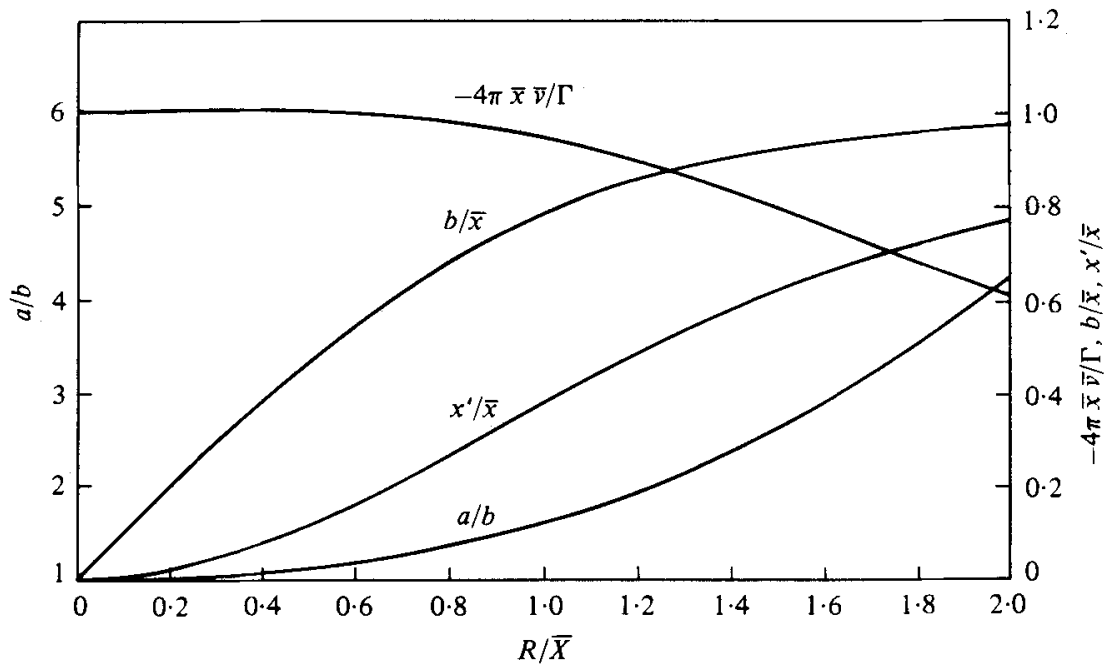

Figure 2. Properties of a vortex pair as functions of $R / \bar{x}$. Each vortex is approximated by an ellipse of major axis $a$ and minor axis $b$ with centres $2 \bar{x}$ apart. $R^{2}=a b . x^{\prime}$ is the displacement of the apparent centre of rotation relative to the centre.

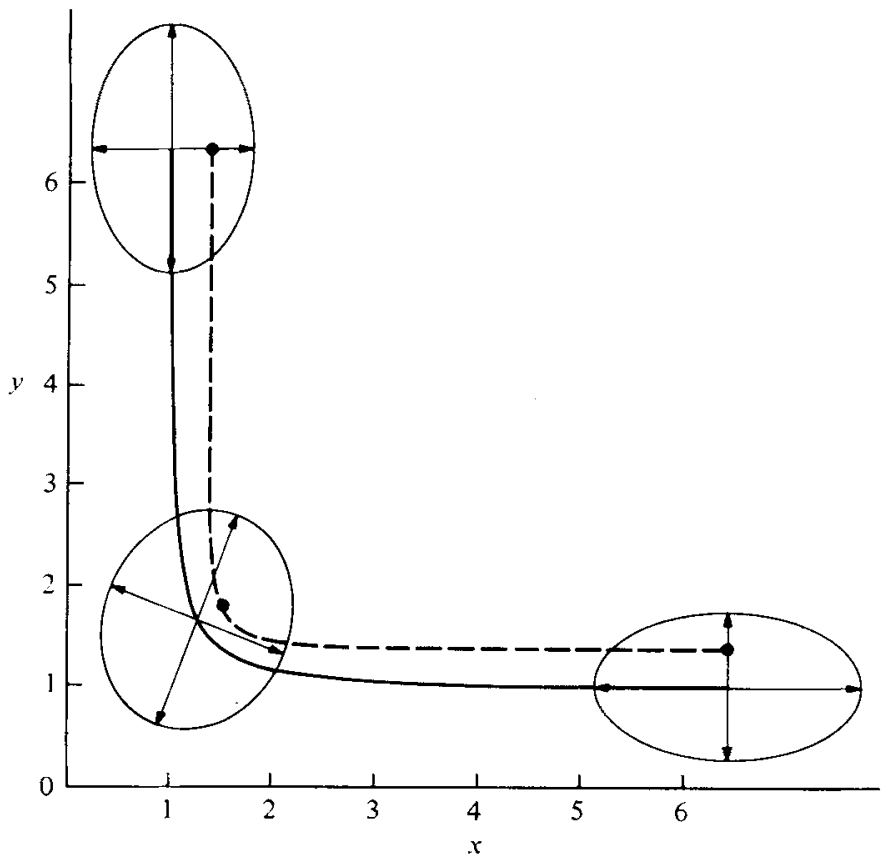

Frgure 3. Approximate calculation of vortex pair in a ground effect for $R / x_{0}=1$. —, path of centre of vortex; ---, path of apparent centre of rotation. Initial, intermediate and final shapes are shown. 9 , position of apparent centre of rotation.

It follows easily that the co-ordinates of the centre of rotation relative to the centre and the $x, y$ axes are

$$
\left.\begin{array}{l}
x^{\prime}=(\pi / \Gamma)(a+b)\left[\bar{u} \sin \theta \cos \theta(a-b)-\bar{v}\left(a \cos ^{2} \theta+b \sin ^{2} \theta\right)\right], \\
y^{\prime}=(\pi / \Gamma)(a+b)\left[\bar{u}\left(a \sin ^{2} \theta+b \cos ^{2} \theta\right)-\bar{v} \sin \theta \cos \theta(a-b)\right] .
\end{array}\right\}
$$


It is assumed in this calculation that the centre of rotation is inside the vortex, which turns out to be the case. The values of $x^{\prime} / x$ are also shown in figure 2 . It will be noted that for distorted vortices, $\epsilon>2$ say, the centre of rotation is displaced from the centroid of vorticity by a significant amount, which might make the vortices appear to be further apart than they actually are.

Results for a vortex pair approaching a wall are shown in figure 3 for $R / x_{0}=1 \cdot 0$. These were obtained by numerical integration of (3.8) using a multistep AdamsMoulton integration scheme with time step $5 x_{0}^{2} / 2 \Gamma$. A modified Euler scheme was used to specify the initial values for the multistep procedure. The values of $\bar{u}$ and $\bar{v}$ were found by solving (3.9) and (3.7) by Newton's method with the values given by table 1 . The integration was completely straightforward. Similar results are found for other values of $R / x_{0}$. For smaller $R / x_{0}$, the deformation of the vortices is reduced and the trajectories of the centroid and apparent centre of rotation are closer. The converse holds for larger $R / x_{0}$. For values of $R / x_{0}$ up to $2 \cdot 0$, for which the axis ratio approximates 4 , the trajectories of the centroids are indistinguishable from those of point vortices. The path of the apparent centre of rotation always approached the wall monotonically. The calculated paths are symmetrical about the line $x=y$ and the approximation does not allow for the existence of oscillations which might occur in reality. In any event, it now seems clear that the explanation of the observed rebounding must be elsewhere than the inviscid dynamics of finite vortices.

The work was supported by the U. S. Army Research Office, Durhen (DAAG 29-78-C-0011) and the Department of Energy (EY-76-S-03-0767).

\section{REF'ER EN CES}

BARKER, S. J. \& Crow, S. C. 1977 The motion of two-dimensional vortex pairs in a ground effect. J. Fluid Mech. 82, 659-671.

Bilanin, A. J., Teske, M. E. \& Williamson, G. G. 1977 Vortex interactions and decay in aircraft wakes. A.I.A.A.J.15, 250-260.

DeE, F. W. \& Nicholas, O. P. 1968 Flight measurements of wing tip vortex motion near the ground. R.A.E. Tech. Rep. no. 68007 .

Harvey, J. K. \& Perry, F. J. 1971 Flowfield produced by trailing vortices in the vicinity of the ground. A.I.A.A.J. 9, 1659-1660.

Hooker, S. G. 1936 On the action of viscosity in increasing the spacing ratio of a vortex sheet. Proc. Roy. Soc. A 154, 67-89.

LAMB, H. 1932 Hydrodynamics, 6th edn. Cambridge University Press.

Moore, D. W. \& SAFFman, P. G. 1971 Structure of a line vortex in an imposed strain. In Aircraft Wake Turbulence (ed. J. H. Olsen, A. Goldburg \& M. Rogers), pp. 339-354. Plenum.

Moore, D. W. \& Safrman, P. G. 1975 The density of organised vortices in a turbulent mixing layer. J. Fluid Mech. 69, 465-473.

Pocklingron, H. C. 1894 The configuration of a pair of equal and opposite hollow straight vortices of finite cross section moving steadily through fluid. Proc. Camb. Phil. Sac. 8, $179-187$. 